\title{
EFEITO DA GESTÃO FAMILIAR NA AVALIAÇÃO DO DESEMPENHO DA EMPRESA: EVIDÊNCIAS DO BRASIL
}

\section{EFFECT OF FAMILY MANAGEMENT IN EVALUATION THE COMPANY'S PERFORMANCE: EVIDENCE FROM BRAZIL}

\author{
ELISANE BRANDT \\ Universidade Regional de Blumenau. Endereço: Rua Antônio da Veiga, \\ 140 / Itoupava Seca | 89030-903 | Blumenau/SC / Brasil. \\ (1) http://orcid.org/0000-0001-8129-0908 \\ E-mail: elisane_brandt@hotmail.com
}

\author{
ADRIANA KROENKE \\ Universidade Regional de Blumenau. Endereço: Rua Antônio da Veiga, \\ 140 / Itoupava Seca | 89030-903 / Blumenau/SC / Brasil. \\ (1) http://orcid.org/0000-0001-6625-3017 \\ E-mail: akroenke@furb.br \\ CAROLINE PLETSCH \\ Universidade Regional de Blumenau. Endereço: Rua Antônio da Veiga, \\ 140 | Itoupava Seca | 89030-903 | Blumenau/SC / Brasil. \\ (1) http://orcid.org/0000-0002-1978-2204 \\ E-mail:carol_spletsch@yahoo.com.br
}

\section{RESUMO}

Esta pesquisa tem como objetivo verificar o efeito da gestão familiar na avaliação do desempenho de empresas brasileiras de capital aberto pertencentes ao setor de consumo cíclico listadas na Brasil, Bolsa, Balcão (B3). Para tanto, realizou-se uma pesquisa descritiva, documental e com abordagem quantitativa por meio da utilização da Regressão Linear Múltipla. O período de análise compreendeu os anos de 2012 a 2016. A amostra do estudo foi constituída por 34 empresas brasileiras familiares e 66 empresas não familiares, totalizando as 100 empresas classificadas no setor de consumo cíclico. Os resultados revelaram diferenças entre as empresas familiares e não familiares quanto ao desempenho contábil, visto que esta variável se relacionou significativa e positivamente com o desempenho medido pela proxy ROA. Isto sugere que as empresas familiares buscam ao longo dos anos melhorar o valor dos ativos, pois quanto maior o desempenho contábil, mais investidores se interessarão nestas empresas, com expectativas de retornos a longo prazo. Outra evidência encontrada é a relação entre o tamanho da empresa e seu desempenho de mercado e contábil. Em empresas familiares, este achado indica que seu tamanho apresenta relação com estas empresas do setor de consumo cíclico, pois tendendo a serem mais eficientes no uso de seus ativos, relacionam-se positivamente com o desempenho. Conclui-se que o desempenho de mercado medido pela proxy $Q$ de Tobin se apresentou como um dos principais indicadores que levam as empresas à uma alta performance organizacional.

Palavras-chave: Empresa Familiar. Desempenho da Empresa. Setor de Consumo Cíclico. Brasil. 


\begin{abstract}
This research aims to verify the effect of family management in performance evaluation of Brazilian publicly traded companies belonging to the cyclical consumer sector listed in Brazil, Bolsa, Balcão (B3). For that, a descriptive, documentary research was conducted with a quantitative approach through the use of Multiple Linear Regression. The period of analysis comprised the years from 2012 to 2016. The study sample consisted of 34 Brazilian family companies and 66 non-family companies, totaling the 100 companies classified in the cyclical consumption sector. The results revealed differences between the family and unfamiliar companies regarding the accounting performance, since this variable was related in a significant and positive way with the performance measured by the ROA proxy. This result suggests that family companies seek over the years to improve the value of assets, since the higher the book performance, the more investors will be interested in family firms with expectations of long-term returns. Other evidence worth mentioning is the relationship between the size of the company and the market and accounting performance of family and non-family companies. In family firms, this finding indicates that the size of the company is related to these companies in the cyclical consumption sector, since they tend to be more efficient in the use of their assets, presenting a positive relation with performance. It is concluded that the market performance, as measured by the Tobin $Q$ proxy, was one of the main measures of performance that lead companies to a high organizational performance.
\end{abstract}

Keywords: Family Business. Performance of the Company. Cyclical Consumption Sector. Brazil.

\title{
1 INTRODUÇÃO
}

As empresas familiares contemplam dois ambientes distintos, a família e o negócio, o que leva estas empresas a buscar constantemente o equilíbrio entre ambos (Beuren, Politelo, \& Martins, 2016; Masri, Tekathen, Magnan, \& Boulianne, 2017). Nesse sentido, suas características peculiares influenciam significativamente os objetivos da empresa e as estratégias implementadas (Chua, Chrisman, \& Sharma, 1999; Erbetta, Menozzi, Corbetta, \& Fraquelli, 2013; Hiebl, Duller, Feldbauer-Durstmüller, \& Ulrich, 2015), distinguindo-as das empresas não familiares.

Para as economias nacionais, as empresas familiares são um dos mais importantes tipos de negócios (Ayranci, 2014; Hiebl et al., 2015; Beuren et al. 2016). De acordo com o Family Firm Institute (2015), no cenário brasileiro $90 \%$ das empresas privadas são familiares, e correspondendo a $50 \%$ do Produto Interno Bruto (PIB) e $85 \%$ dos empregos gerados. Estas contudo se distinguem das demais empresas por possuírem uma base de envolvimento familiar na condução dos negócios (Donckels \& Frochich, 1991; Chua et al., 1999; Shyu, 2011).

Por conseguinte, padrões de propriedade, governança, gestão e sucessão influenciam significativamente os objetivos da empresa familiar e as estratégias implementadas (Chua et al., 1999; Mazzi, 2011). Gómez-Mejía, Haynes, Núñez-Nickel, Jacobson, \& Moyano-Fuentes (2007) apontam como vantagens potenciais destas o comprometimento organizacional a longo prazo, visto que preocupam-se com a riqueza contida na perpetuação dos valores da família por meio do negócio (Chua et al., 1999; Shyu, 2011) e ao longo das gerações (Reyna \& Encalada, 2016), o que reflete no desempenho de mercado e contábil das empresas familiares.

Nestas empresas, os laços familiares podem criar condições para a obtenção de um desempenho diferenciado em comparação com as não familiares (Martínez, Stöhr \& Quiroga, 2007; Shyu, 2011; Erbetta et al., 2013). Embora haja evidências na literatura da influência familiar no desempenho das empresas (Sharma, Chrisman, \& Chua, 1997; Anderson \& Reeb, 2003; Martínez et al., 2007; Amran \& Ahmad, 2009; Bonilla, Sepulveda \& Carvajar, 2010; Shyu, 2011; Din \& Javid, 2011; Mazzi, 2011; Speckbacher \& Wentges, 2012; Erbetta et al., 2013; Hamberg, Fagerland, \& Nilsen, 2013; Pukthuanthong, Walker, Thiengtham, \& Du, 2013; Muttakin, Khan, \& Subramaniam, 2014; Ayranci, 2014; Vieira, 2014; Politelo, Kaveski, Chiarello, \& Silva, 2014; Halili, Saleh, \& Zeitun, 2015; Hiebl et al., 2015; Beuren et al., 2016; Kang \& Kim, 2016; PolettiHughes \& Williams, 2017; Saleh, Halili, Zeitun \& Salim, 2017; Costa, Macedo, Yokoyama \& Almeida, 2017), tal configuração merece ser melhor explorada, visto que a presença de familiares 
na gestão implica em melhores desempenhos e sustentação da vantagem competitiva (Mazzi, 2011; Speckbacher \& Wentges, 2012). Além disso, a família pode exercer influência e controle na gestão da empresa, ter envolvimento direto nas decisões, tanto estratégicas quanto operacionais (Gómez-Mejía et al., 2007; Sciascia, Mazzola \& Kellermanns, 2014).

De acordo com Pukthuanthong et al. (2013), apesar da literatura existente, a relação da gestão familiar com os desempenhos de mercado e contábil permanece em grande parte sem resposta, dados os resultados contraditórios acerca de seu impacto no valor da empresa. Estes fatores estimulam o desenvolvimento desta pesquisa, que vislumbra uma lacuna ainda não preenchida na área de finanças corporativas. Além disso, as empresas familiares costumam ter avaliações de desempenho e lucratividade mais altas do que as não familiares (Anderson \& Reeb, 2003), e ainda não há na literatura evidências sobre a influência do ciclo de vida no desempenho das primeiras, revelando outra oportunidade para este estudo. No que tange este último aspecto, Franks, Mayer, Volpin e Wagner (2012) encontraram evidências de que, em países com forte proteção ao investidor e mercados financeiros desenvolvidos, as empresas familiares evoluem ao longo de seu ciclo de vida para configurações capital aberto à medida que amadurecem, enquanto em países com fraca proteção ao investidor e mercados financeiros menos desenvolvidos, o controle familiar é intenso e duradouro.

Nesse contexto, considerando a importância das empresas familiares para a economia nacional, dadas sua representatividade e suas particularidades, este estudo busca esclarecer o efeito da gestão familiar no desempenho das empresas brasileiras de capital aberto pertencentes ao setor de consumo cíclico listadas na Brasil, Bolsa, Balcão (B3).

Conforme Chahine (2007), embora vantajoso, o papel do controle familiar é pouco explorado nas finanças corporativas, sendo necessárias pesquisas que visam analisar a ligação entre empresa familiar e seu desempenho, visto que esta relação é complexa (Mazzi, 2011). Buscando elucidar os aspectos dessa relação, este estudo também se justifica por conta de as empresas familiares serem a forma mais comum de organização no mundo e, no geral, o controle familiar pode ter um efeito positivo no desempenho empresarial (Kang \& Kim, 2016).

Dada a representatividade das empresas familiares na economia mundial (Zahra \& Sharma, 2004), assim como seu impacto econômico significativo (Speckbacher \& Wentges, 2012), é inegável a importância dessas empresas para as esferas sociais e culturais (Acquaah, 2013). Além disso, por conta de o Brasil ser um país emergente, as empresas familiares nacionais têm notória participação na economia, superior aos países desenvolvidos (Politelo et al., 2014), fatores que também justificam a elaboração desta pesquisa.

No geral, este trabalho contribui para a literatura sobre os desempenhos de mercado e contábil, pois se concentra no impacto das decisões familiares nestes indicadores, considerando novas variáveis independentes como determinantes do desempenho, como o ciclo de vida das organizações. Assim, os resultados empíricos deste estudo fornecem informações úteis para pesquisadores, profissionais e investidores que operam no mercado de capitais, no que tange empresas familiares e não familiares (Saleh et al., 2017).

\section{DESEMPENHO DAS EMPRESAS FAMILIARES}

Devido à predominância e representatividade dos negócios familiares, e seu significativo impacto econômico (Speckbacher \& Wentges, 2012) tanto em países desenvolvidos quanto em desenvolvimento, estudos acerca da teoria, pesquisa e prática dessa área vêm se intensificando ao longo dos últimos anos (Zahra \& Sharma, 2004; Speckbacher \& Wentges, 2012; Dawson \& Mussolino, 2014). Contudo ainda é recorrente na literatura a ambiguidade de definições de empresas familiares, devido a suas especificidades (Chua et al., 1999; Shyu, 2011).

A característica familiar dessas empresas pode, em certa medida, afetar seu desempenho, visto que o conselho da maioria delas é formado por membros da família. Assim, indiretamente, essa configuração dos conselhos pode afetar o desempenho da empresa (Amran \& Ahmad, 2009). Além disso, muitas empresas de capital aberto são possuídas e controladas por suas famílias fundadoras (Burkart, Panunzi \& Shleifer, 2003; Hamberg et al., 2013), sendo uma das formas mais prevalentes de estrutura de propriedade em todo o mundo, contribuindo fortemente para suas economias (Pukthuanthong et al., 2013). Deste modo, uma das principais características das empresas familiares é a influência da família na gestão da empresa, seja 
direta (administração geral está sob a responsabilidade familiar), ou indireta (a família não faz parte da gestão da empresa, mas integra o conselho de administração ou participa de decisões administrativas) (Beuren, Hein \& Boff, 2011). Nesse sentido, o desempenho das empresas é um elemento importante da gestão, uma vez que reflete em sua competitividade (Beuren, Politelo, \& Martins, 2013).

Assim, o envolvimento familiar está associado a uma forma específica de gerir e controlar as operações das empresas (Ussman, 1996; Zahra, 2005; Holt, Rutherford, \& Kuratko, 2010), o que pode apresentar reflexos positivos no desempenho das empresas familiares. Por consequência, e devido à intensidade do envolvimento familiar, as empresas criam um conjunto específico de objetivos que contemplam os interesses e valores familiares (Ussman, 1996; Sharma et al., 1997; Gómez-Mejia et al., 2007; Shyu, 2011), por sua vez utilizados para perseguir a visão da empresa (Donckels \& Frochich, 1991; Chua et al., 1999; Sciascia et al ., 2014) e atingir uma combinação de metas financeiras e não financeiras, ou seja, o desempenho de mercado e contábil almejado (Sharma, 2004; Poletti-Hoghes \& Williams, 2017).

Deste modo, a participação familiar se concentra na necessidade de exercer autoridade e controle na organização (Berrone, Cruz \& Gomez-Mejia, 2012), bem como interferir no desempenho da empresa. De acordo com Speckbacher e Wentges (2012), as empresas familiares apresentam desempenhos melhores que as não familiares, e consequentemente detêm vantagem competitiva (Mazzi, 2011). Nesse sentido, em comparação com empresas não familiares, as organizações familiares, devido a suas características peculiares, podem apresentar um desempenho econômico-financeiro diferenciado (Erbetta et al., 2013).

O desempenho das empresas familiares é condizente com a performance em termos das dimensões empresariais e familiares, considerando-se qualquer ponto de seu ciclo de vida (Sharma, 2004). Speckbacher e Wentges (2012) mencionam a tendência conservadora das empresas familiares no planejamento estratégico nos primeiros anos de vida. No entanto, com o passar do tempo tornam-se mais agressivas devido ao ambiente competitivo. Conforme Franks et al. (2012), o controle familiar é importante pois domina diversos mercados financeiros em todo o mundo e, de acordo com a visão do ciclo de vida, os determinantes do controle familiar interagem com a idade da empresa, ou seja, com o ciclo de vida que esta se encontra (Franks et al., 2012), denotando a relação entre ambos os fatores.

A pesquisa desenvolvida por Speckbacher e Wentges (2012) revelou que as empresas que possuem membros fundadores atuando como executivos em geral possuem um desempenho melhor do que as demais. Contudo, diferentes estratégias levam ao uso de diferentes tipos de indicadores de desempenho (Micheli \& Mura, 2017). Assim, o desempenho se diferencia nas empresas familiares e não familiares. Outro delimitador do uso de indicadores financeiros advém do estágio do ciclo de vida que a empresa se encontra, ou seja, as alterações que a empresa sofre ao longo do tempo, como mudanças organizacionais, padrões dos sistemas de controle gerenciais, caracterizados por estágios de desenvolvimento que podem afetar seu desempenho (Dickinson, 2011; Costa et al., 2017), além do modo como a organização, seja familiar ou não, enfrenta os desafios de gestão.

A avaliação do desempenho das empresas tem se destacado na gestão das organizações, pois seja contábil ou de mercado, é fundamental para sua sobrevivência (Macedo \& Corrar, 2010), tornando a análise deste de suma importância, por ser essencial para o controle gerencial dos negócios (Olson \& Slater, 2002). Deste modo, se faz necessário o entendimento das variáveis que influenciam o desempenho das empresas familiares e não familiares, conforme proposto nesta pesquisa. No entanto, apesar da relevância da avaliação do desempenho das empresas na literatura, esta temática tem sido objeto de discussão, principalmente no que se refere ao uso de indicadores (Macedo \& Corrar, 2010).

Nos estudos revisitados e desenvolvidos a partir de empresas familiares e não familiares, geralmente foram analisados indicadores contábeis como o Retorno sobre os Ativos (ROA), o Retorno sobre o Patrimônio Líquido (ROE), o Market-to-Book, bem como o $Q$ de Tobin, para identificar também o desempenho de mercado.

A pesquisa desenvolvida por Anderson e Reeb (2003) evidenciou que as empresas familiares apresentam um $Q$ de Tobin e um ROA mais elevados, quando comparadas às não familiares. Assim, a participação dos familiares na gestão das empresas reflete no desempenho destas. No que diz respeito à maior lucratividade, Maury (2006) investigou empresas familiares 
e não familiares da Europa Ocidental, revelando uma maior lucratividade das primeiras em comparação com empresas não familiares.

O estudo de Andres (2008) analisou o desempenho de empresas familiares da Alemanha, indicando que estas são mais lucrativas e detém ações principais no mercado financeiro, quando comparadas às demais empresas pesquisadas. Contudo, o desempenho das empresas familiares apenas é melhor naquelas em que a família fundadora ainda está ativa no conselho executivo ou fiscal. Nesse sentido, evidencia-se que a propriedade familiar se relaciona com maior desempenho da empresa apenas sob estas condições. Além disso, quando as famílias são apenas grandes acionistas, sem representação significante nos conselhos, o desempenho dessas empresas não se distingue das demais.

Din e Javid (2011) investigaram a influência do envolvimento familiar no desempenho de empresas de capital aberto do Paquistão, por meio das variáveis de desempenho contábil ROA e ROE. Conforme os resultados aferidos, existe uma relação positiva e significante entre os fatores determinantes de empresa familiar e seu desempenho. O estudo de Shyu (2011) também teve como objetivo avaliar a influência da propriedade familiar no desempenho das empresas de capital aberto de Taiwan, revelando que o desempenho medido pelas proxies $R O A$ e $Q$ de Tobin se relacionou positivamente com a propriedade familiar. O autor concluiu então que em empresas familiares há um engajamento maior na obtenção de melhores desempenhos.

Erbetta et al. (2013) também obtiveram uma relação positiva entre a propriedade familiar e o desempenho de suas empresas. Ayranci (2014) desenvolveu uma pesquisa utilizando em sua amostra empresas familiares e não familiares, destacando que a influência da família sobre o negócio deve ser considerado por meio das análises do desempenho destas organizações. Reyna e Encalada (2016) analisaram o efeito da sucessão no desempenho econômicofinanceiro, pontuando que, em um processo de mudança da segunda para a terceira geração familiar, há um efeito positivo sobre o indicador em questão.

Diante dos contextos mencionados, denota-se que a gestão familiar pode desencadear efeitos positivos no desempenho das empresas familiares, o que estimula o desenvolvimento desta pesquisa no cenário brasileiro de empresas de capital aberto. Além disso, a relevância dos trabalhos apresentados é evidente, visto que revelam o diferencial das empresas familiares em seu desempenho. Assim, conforme Beuren et al. (2016), as diferentes perspectivas das famílias, no que diz respeito aos investimentos em empresas, resultam no desempenho de suas empresas, distinguindo-as das demais por suas particularidades que, consequentemente, apresentam reflexos no desempenho. Na sequência, apresentam-se os procedimentos adotados para a realização desta pesquisa.

\section{PROCEDIMENTOS METODOLÓGICOS}

A fim de verificar o efeito da gestão familiar no desempenho de empresas brasileiras, esta pesquisa se caracteriza como descritiva; quanto aos procedimentos, como documental e; no que tange a abordagem do problema, como quantitativa, visto que este estudo utilizou métodos estatísticos para evidenciar os resultados.

\subsection{População e Amostra}

A população do estudo é composta pelas 100 empresas brasileiras familiares e não familiares de capital aberto listadas na B3, classificadas no setor de consumo cíclico. Para tanto, a amostra foi composta por 34 empresas familiares e as demais não familiares, com vistas à comparação dos resultados.

Optou-se pelo setor de consumo cíclico por este possuir a maior porcentagem de empresas caracterizadas como familiares (Beuren et al., 2013). Além disso, esta amostra possui relevância no cenário brasileiro, pois contribui para cerca de $50 \%$ do PIB e $85 \%$ dos empregos gerados (Family Firm Institute, 2015), gerando um impacto econômico significativo (Speckbacher \& Wentges, 2012). Assim, são partes integrantes da base do crescimento econômico e desenvolvimento aferido no país, sendo de extrema importância para as esferas econômicas, sociais e culturais (Acquaah, 2013). Optou-se ainda por analisar empresas familiares cotadas em bolsa de valores, pois conforme Vieira (2014), ao longo das décadas, diversos estudos 
evidenciaram que as empresas familiares estão presentes entre as empresas de capital aberto em todo o mundo.

Ainda segundo Vieira (2014), uma dificuldade neste tipo de estudo é a definição de empresas familiares e não familiares. Para esta pesquisa, esta identificação foi feita por meio do Formulário de Referência disponível no site da Comissão de Valores Mobiliários (CVM), no item 15.1/2, para a participação familiar no capital, e nos itens 12.6/8 e 12.9, para a participação familiar na empresa. Assim, definiu-se como familiares as empresas que apresentaram a concentração de propriedade com uma família e/ou a participação dos membros da família no conselho de administração (Shyu, 2011; Politelo et al., 2014).

Além disso, para determinar a empresa como familiar utilizou-se um percentual de concentração mínimo de ações de 10\% (Anderson \& Reeb, 2003; Shyu, 2011; Politelo et al., 2014). No entanto, há diferentes combinações entre propriedade e/ou gestão familiar possíveis, ao se considerar a complexidade das organizações (Frezatti, Bido, Mucci, \& Beck, 2017). De acordo com Chua et al. (1999), estas combinações são três: propriedade e gestão familiar; propriedade não familiar e gestão familiar; propriedade familiar e gestão não familiar.

\subsection{Coleta e Análise dos Dados}

A coleta de informações se deu na base de dados Economática ${ }^{\circledR}$, nos Formulários de Referência divulgados no site da B3 ou nos próprios sites das empresas, anualmente, no período compreendido entre os anos de 2012 a 2016. A especificidade do período de amostragem resulta da disponibilidade de dados. De acordo com os estudos anteriores revisitados nesta pesquisa, utilizou-se três variáveis dependentes e três independentes para explicar o desempenho das empresas familiares e não familiares, que são apresentadas na Tabela 1.

Tabela 1

Variáveis da pesquisa

\begin{tabular}{|c|c|c|c|}
\hline Classificação & Variável & Mensuração & Autores (Ano) \\
\hline \multirow{3}{*}{ 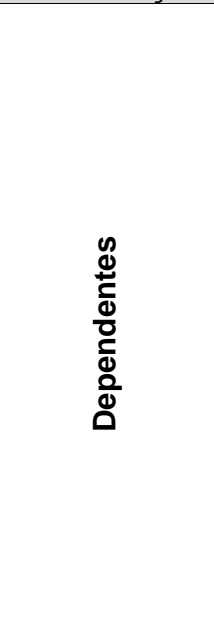 } & $Q$ de Tobin (QT)* & $\frac{\text { VMA + D }}{\text { Ativo Total }}$ & $\begin{array}{l}\text { Anderson e Reeb (2003); Martínez et al. (2007); } \\
\text { Amran e Ahmad (2009); Shyu (2011); Erbetta et al. } \\
\text { (2013); Hamberg et al. (2013); Pukthuanthong et } \\
\text { al. (2013); Muttakin et al. (2014); Vieira (2014); } \\
\text { Beuren, Politelo e Martins (2016); Poletti-Hughes e } \\
\text { Williams (2017). }\end{array}$ \\
\hline & $\begin{array}{l}\text { Retorno sobre o } \\
\text { Ativo Total (ROA) }\end{array}$ & $\frac{\text { Lucro Operacional }}{\text { Ativo Total }}$ & $\begin{array}{l}\text { Anderson e Reeb (2003); Martínez et al. (2007); } \\
\text { Amran e Ahmad (2009); Bonilla et al. (2010); Shyu } \\
\text { (2011); Dickinson (2011); Pukthuanthong et al. } \\
\text { (2013); Muttakin et al. (2014); Vieira (2014); Halili } \\
\text { et al. (2015); Beuren et al. (2016); Saleh et al. } \\
\text { (2017). }\end{array}$ \\
\hline & $\begin{array}{l}\text { Retorno sobre o } \\
\text { Patrimônio } \\
\text { Líquido (ROE) }\end{array}$ & $\frac{\text { Lucro Líquido }}{\text { Patrimônio Líquido }}$ & $\begin{array}{l}\text { Martínez et al. (2007); Bonilla et al. (2010); Vieira } \\
\text { (2014); Halili et al. (2015); Saleh et al. (2017); } \\
\text { Costa et al. (2017). }\end{array}$ \\
\hline 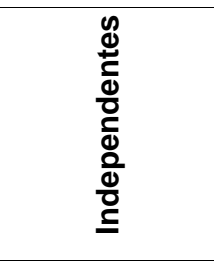 & $\begin{array}{l}\text { Empresa Familiar } \\
\text { (EF) }\end{array}$ & $\begin{array}{l}\text { Dummy: (Atribuiu-se } 1 \\
\text { para empresa familiar e } 0 \\
\text { para empresa não } \\
\text { familiar) }\end{array}$ & $\begin{array}{l}\text { Anderson e Reeb (2003); Amran e Ahmad (2009); } \\
\text { Ayranci (2014); Speckbacher e Wentges (2012); } \\
\text { Hamberg et al. (2013); Pukthuanthong et al. } \\
\text { (2013); Politelo et al. (2014); Muttakin et al. (2014); } \\
\text { Vieira (2014); Halili et al. (2015); Hiebl et al (2015); } \\
\text { Beuren et al. (2016); Kang; Kim (2016); Poletti- } \\
\text { Hughes e Williams (2017); Saleh et al. (2017). }\end{array}$ \\
\hline
\end{tabular}

Continua 
Tabela 1 (continuação)

\begin{tabular}{|c|c|c|c|}
\hline Classificação & Variável & Mensuração & Autores (Ano) \\
\hline \multirow{3}{*}{ 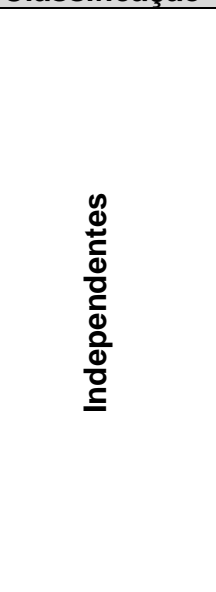 } & Ciclo de Vida (CV) & $\begin{array}{l}\text { Conforme Tabela 2, } \\
\text { atribuiu-se: } \\
\text { (1) Nascimento; } \\
\text { (2) Crescimento; } \\
\text { (3) Maturidade; } \\
\text { (4) Turbulência; } \\
\text { (5) Declínio. }\end{array}$ & $\begin{array}{c}\text { Dickinson (2011); Costa et al. (2017); Frezatti et } \\
\text { al. (2017). }\end{array}$ \\
\hline & \multicolumn{3}{|c|}{ Variável de Controle } \\
\hline & Tamanho (TAM) & Log (Ativo Total) & $\begin{array}{l}\text { Anderson e Reeb (2003); Martínez et al. (2007); } \\
\text { Amran e Ahmad (2009); Bonilla et al. (2010); Shyu } \\
\text { (2011); Speckbacher e Wentges (2012); Hamberg } \\
\text { et al. (2013); Halili et al. (2015); Hiebl et al. (2015); } \\
\text { Beuren et al. (2016); Kang e Kim (2016); Poletti- } \\
\text { Hughes e Williams (2017); Costa et al. (2017). }\end{array}$ \\
\hline
\end{tabular}

Nota. Legenda: * VMA = Valor de mercado das ações negociadas em bolsa (obtido a partir da multiplicação do número de ações da companhia pelo seu preço contado em bolsa de valores); D = VCPC - VCAC + VCE + VCDLP (VCPC: Valor contábil dos passivos circulantes da empresa; VCAC: Valor contábil dos ativos circulantes da empresa; VCE: Valor contábil dos estoques; VCDLP: Valor contábil das dívidas de longo prazo).

Fonte: Elaboração própria (2018).

Nesta pesquisa consideraram-se medidas contábeis (ROA e ROE) e de mercado ( $Q$ de Tobin) como proxies para verificar se há diferenças no desempenho das empresas, ou seja, o efeito familiar e não familiar neste indicador.

Um dos diferenciais deste estudo é a utilização da variável explicativa ciclo de vida, pois o estágio em que a empresa se encontra reflete no desempenho desta. Para tanto, a variável em questão foi categorizada conforme as definições desenvolvidas por Dickinson (2011), as quais são apresentadas na Tabela 2.

\section{Tabela 2}

\section{Estágio do Ciclo de Vida conforme modelo de Dickinson (2011)}

\begin{tabular}{|c|c|c|c|c|c|}
\hline Fases & Nascimento & Crescimento & Maturidade & Turbulência & Declínio \\
\hline \multirow[t]{2}{*}{ 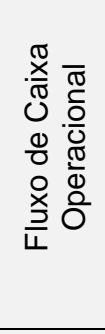 } & $\begin{array}{c}\text { As empresas entram } \\
\text { no mercado com } \\
\text { déficit de } \\
\text { conhecimentos } \\
\text { sobre receitas e } \\
\text { custos. }\end{array}$ & $\begin{array}{l}\text { As margens de lucro } \\
\text { são maximizadas } \\
\text { durante o período de } \\
\text { maior investimento. }\end{array}$ & $\begin{array}{c}\text { A eficiência } \\
\text { maximizada por } \\
\text { meio do aumento de } \\
\text { conhecimentos } \\
\text { operacionais. }\end{array}$ & $\begin{array}{c}\text { O declínio do } \\
\text { crescimento leva à } \\
\text { queda dos preços. } \\
\text { As rotinas } \\
\text { estabelecidas } \\
\text { impedem a } \\
\text { flexibilidade. }\end{array}$ & $\begin{array}{l}\text { O declínio do } \\
\text { crescimento leva a } \\
\text { queda dos preços. }\end{array}$ \\
\hline & Fluxo de Caix & Fluxo de Caix & Fluxo de Caixa (+) & Fluxo de Caixa (+/-) & Fluxo de Caixa (-) \\
\hline \multirow[t]{2}{*}{ 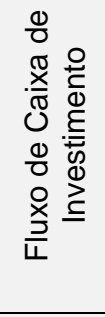 } & $\begin{array}{l}\text { Otimismo gerencial } \\
\text { impulsiona o } \\
\text { investimento. }\end{array}$ & $\begin{array}{l}\text { As empresas fazem } \\
\text { grandes } \\
\text { investimentos } \\
\text { antecipados para } \\
\text { impedir a entrada. }\end{array}$ & $\begin{array}{c}\text { A obsolescência } \\
\text { aumenta em relação } \\
\text { ao novo } \\
\text { investimento. }\end{array}$ & Vazio em teoria. & $\begin{array}{l}\text { Liquidação de ativos } \\
\text { para amortizar } \\
\text { dívidas. }\end{array}$ \\
\hline & Fluxo de Caixa (-) & Fluxo de Caixa (-) & Fluxo de Caixa (-) & Fluxo de Caixa ( & Fluxo de Caixa (+) \\
\hline \multirow[t]{2}{*}{ 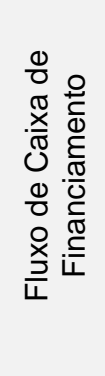 } & $\begin{array}{c}\text { Empresas em } \\
\text { crescimento } \\
\text { acessam a dívida } \\
\text { bancária, ou seja, } \\
\text { aumentam a dívida. }\end{array}$ & $\begin{array}{c}\text { Empresas em } \\
\text { crescimento } \\
\text { acessam a dívida } \\
\text { bancária, aumentam } \\
\text { a dívida. }\end{array}$ & $\begin{array}{l}\text { Mudança de foco } \\
\text { para aquisição de } \\
\text { financiamento e } \\
\text { distribuição de } \\
\text { dividendos em } \\
\text { excesso para os } \\
\text { acionistas, de modo } \\
\text { que as empresas } \\
\text { diminuem a dívida. }\end{array}$ & Vazio em teoria. & $\begin{array}{c}\text { Foco no reembolso } \\
\text { e/ou negociação da } \\
\text { dívida. }\end{array}$ \\
\hline & Fluxo de Caixa (+) & Fluxo de Caixa (+) & Fluxo de Caixa (-) & Fluxo de Caixa (+/-) & Fluxo de Caixa (+/-) \\
\hline
\end{tabular}

Fonte: Adaptado de Dickinson, V. Cash flow pattems as a proxy for firm life cycle. The Accounting Review, 86(6),1969-1994. (2011) 
Para identificar o estágio do ciclo de vida das empresas, esta pesquisa se baseou no estudo de Dickinson (2011), que demonstrou como as combinações dos fluxos de caixa relacionados a operações da empresa, seus investimentos e financiamentos podem ser utilizadas para classificar o estágio do ciclo de vida das organizações, e como estas fases explicam a lucratividade das empresas. Nesse sentido, optou-se pelo modelo de identificação proposto pelo autor por este se alinhar com os propósitos deste estudo.

O estágio de nascimento da empresa foi classificado com o número 1; de crescimento com o número 2; maturidade, número 3; posteriormente, quando a empresa se apresenta em turbulência, classificou-se com o número 4 e, por fim, o número 5 foi destinado para as empresas em declínio. Para tanto, conforme a Tabela 2, a identificação das fases do ciclo de vida das empresas se deu por análise vertical. Em outras palavras, a empresa foi classificada na fase de nascimento quando apresentou, nos anos analisados, fluxos de caixa operacional e de investimento negativos e de financiamento, positivo. Para a fase de crescimento, a empresa apresenta fluxos de caixa positivo para operacional, negativo para investimento e positivo para financiamento. $\mathrm{Na}$ fase de maturidade, o fluxo de caixa apresenta valores positivos para operacional e negativos para investimento e financiamento. A fase da turbulência é identificada pelos três fluxos de caixa positivos ou negativos. Por fim, a fase de declínio pode ser identificada por meio do fluxo de caixa negativo para operacional, positivo para investimento e positivo ou negativo para o financiamento.

Para a análise dos dados realizou-se a Regressão Linear Múltipla por meio do software SPSS $^{\circledR}$. Optou-se por este método pois, de acordo com Wooldridge (2012), ele possibilita analisar uma série de tempo para cada corte transversal do conjunto de dados e passa a eliminar os efeitos das variáveis que foram excluídas a partir do estudo de variações na variável dependente ao longo dos anos analisados. Este método oferece também a possibilidade de estudar a relação entre uma ou mais variáveis explicativas (Fávero, 2015). O modelo de regressão desta pesquisa é definido da seguinte maneira:

\section{Desempenho $=\beta_{0}+\beta_{1}$ Empresa Familiar $+\beta_{2} \mathrm{CV} 1+\beta 3 \mathrm{CV} 2+\beta 4 \mathrm{CV} 3+\beta 5 \mathrm{CV} 4+\beta 6 \mathrm{CV} 5+\beta_{7}$ Tamanho $+\varepsilon$}

Ressalta-se que inicialmente considerou-se como variável dependente (desempenho) no modelo o $Q$ de Tobin, posteriormente o ROA e, por fim, o ROE. Posteriormente apresenta-se a análise dos dados e os resultados obtidos para as empresas familiares e não familiares brasileiras abordadas neste estudo.

\section{DESCRIÇÃO E ANÁLISE DOS DADOS}

De acordo com os procedimentos elencados na metodologia, realizou-se uma análise descritiva das empresas pertencentes à amostra. Na sequência, apresenta-se a matriz de correlação para evidenciar a relação entre as variáveis dependentes e independentes. Por fim, uma análise de regressão foi conduzida para observar se há efeito da gestão familiar no desempenho das empresas estudadas. Primeiramente analisou-se as empresas familiares e não familiares conjuntamente (variável dicotômica).

$\mathrm{Na}$ Tabela 3 são apresentadas as estatísticas descritivas das variáveis dependentes e independentes. Ressalta-se que não foram consideradas as variáveis Empresa Familiar (EF) e Ciclo de Vida (CV) para a estatística descritiva, visto serem variáveis dicotômicas.

\section{Tabela 3}

\section{Estatística descritiva das variáveis}

\begin{tabular}{l|c|c|c|c}
\hline \multicolumn{1}{c|}{ Variável } & Mínimo & Máximo & Média & Desvio-padrão \\
\hline QT & 3,006 & 8,472 & 5,856 & 0,814 \\
\hline ROA & 0,000 & 0,814 & 0,083 & 0,095 \\
\hline ROE & 0,000 & 2,835 & 0,472 & 2,134 \\
\hline TAM & 10,250 & 17,530 & 14,143 & 1,492 \\
\hline
\end{tabular}

Fonte: Dados da pesquisa. 
Observa-se na Tabela 3 que a amostra é composta por um grupo de empresas heterogêneas, embora sejam classificadas no mesmo setor. Constata-se que há empresas com desempenhos favoráveis, e em contraponto, há empresas com baixo desempenho. No geral, tanto as empresas familiares quanto as não familiares possuem desempenho equiparado, embora a variável Retorno sobre o Patrimônio Líquido apresente valores máximos e médios que indiquem a presença de algumas empresas com melhor ROE que outras. Assim, as características da amostra revelam empresas com alto e baixo desempenho contábil e de mercado, rentabilidade, bem como tamanho.

Antes de analisar o efeito da gestão familiar na avaliação de desempenho das empresas brasileiras de capital aberto pertencentes ao setor de consumo cíclico listadas na B3 por meio da Regressão Linear Múltipla, realizou-se os testes de normalidade e a correlação de Spearman entre as variáveis para identificar possíveis problemas de multicolinearidade, conforme apresentado nas Tabelas 4 e 5.

Tabela 4

\section{Teste de Normalidade}

\begin{tabular}{l|c|c|c|c|c|c}
\hline \multirow{2}{*}{ Variável } & \multicolumn{3}{c|}{ Kolmogorov-Smirnov } & \multicolumn{3}{c}{ Shapiro-Wilk } \\
\cline { 2 - 7 } & Estatística & Df & Sig. & Estatística & Df & Sig. \\
\hline QT & 0,167 & 344 & 0,000 & 0,797 & 344 & 0,000 \\
\hline EF & 0,418 & 344 & 0,000 & 0,135 & 344 & 0,000 \\
\hline ROA & 0,209 & 344 & 0,000 & 0,639 & 344 & 0,000 \\
\hline ROE & 0,068 & 344 & 0,001 & 0,976 & 344 & 0,000 \\
\hline CV & 0,252 & 344 & 0,000 & 0,890 & 344 & 0,000 \\
\hline TAM & 0,344 & 344 & 0,000 & 0,636 & 344 & 0,000 \\
\hline
\end{tabular}

Fonte: Dados da pesquisa (2018).

Os dados apresentados na Tabela 4 não são normais, visto que os testes de KolmogorovSmirnov e Shapiro-Wilk apresentaram significância ao nível de 5\%, indicando sua distribuição fora do padrão. Em seguida efetuou-se a correlação de Spearman para identificar a intensidade e relação entre as variáveis e os possíveis problemas de multicolineariedade (Fávero, Belfiore, Chan \& Silva, 2009). Este teste não sugere apenas uma relação de causa e efeito, mas também de associação entre as variáveis estudadas. Segundo Gujarati e Porter (2006), a presença de multicolinearidade nos dados não significa que o modelo apresenta problemas, no entanto, quando elevada em relação às variáveis, esta aumenta consequentemente o erro. Na Tabela 5 evidenciam-se os resultados da correlação de Spearman entre as variáveis analisadas na pesquisa.

\section{Tabela 5}

\section{Correlação de Spearman entre as variáveis}

\begin{tabular}{l|c|c|c|c|c|c}
\hline Variáveis & ROA & ROE & QT & TAM & CV & EF \\
\hline ROA & 1 & 0,585 & $-0,234$ & 0,229 & $0,002^{* *}$ & 0,150 \\
\hline ROE & & 1 & $0,010^{*}$ & $0,055^{\star *}$ & $0,022^{* *}$ & $0,038^{* *}$ \\
\hline QT & & & 1 & $-0,845$ & 0,175 & 0,039 \\
\hline TAM & & & & 1 & $-0,077$ & $-0,124$ \\
\hline CV & & & & & 1 & $0,021^{* *}$ \\
\hline EF & & & & & & 1 \\
\hline
\end{tabular}

Nota. Legenda: * A correlação é significativa no nível 1\%. ${ }^{*}$ A correlação é significativa no nível $5 \%$.

Fonte: Dados da pesquisa (2018).

Conforme os dados apresentados na Tabela 5, nota-se a existência de relação entre as variáveis dependentes e as independentes. Deste modo, não há alto grau de correlação entre as variáveis explicativas (correlação superior a 85\%), o que revela não haver problemas de multicolinearidade que afetem os resultados do modelo de regressão com as variáveis utilizadas. Assim, todas as variáveis explicativas ilustram o efeito da gestão familiar no desempenho das empresas brasileiras de capital aberto listadas na B3 pertencentes ao setor de consumo cíclico. Estes resultados revelam que o envolvimento familiar se relaciona com as demais variáveis do estudo, embora, isto seja melhor explicado por meio da análise de Regressão Linear Múltipla, 
que utilizando como variável dependente o $Q$ de Tobin, e na sequência, o ROA e o ROE, resultou em três modelos, apresentados na Tabela 6.

Tabela 6

Resultados do modelo da Regressão Linear

\begin{tabular}{|c|c|c|c|c|}
\hline \multicolumn{2}{|c|}{ Variável } & Q de Tobin & ROA & ROE \\
\hline \multirow{2}{*}{ Constante } & Coeficiente & $-5,80$ & 3,37 & $-0,32$ \\
\hline & Sig. & $0,00^{*}$ & $0,00^{*}$ & 0,75 \\
\hline \multirow{2}{*}{ Empresa Familiar } & Coeficiente & $-0,01$ & 3,33 & $-0,88$ \\
\hline & Sig. & 0,996 & $0,00^{*}$ & 0,38 \\
\hline \multirow{2}{*}{ Nascimento } & Coeficiente & $-2,37$ & $-3,12$ & $-1,82$ \\
\hline & Sig. & $0,02^{*}$ & $0,00^{*}$ & $0,07^{\star *}$ \\
\hline \multirow{2}{*}{ Crescimento } & Coeficiente & $-1,88$ & $-0,41$ & $-0,08$ \\
\hline & Sig. & $0,06^{\star *}$ & 0,68 & 0,94 \\
\hline \multirow{2}{*}{ Turbulência } & Coeficiente & $-0,26$ & $-1,33$ & $-0,08$ \\
\hline & Sig. & 0,79 & 0,19 & 0,93 \\
\hline \multirow{2}{*}{ Declínio } & Coeficiente & $-0,73$ & $-1,60$ & $-0,21$ \\
\hline & Sig. & 0,47 & 0,11 & 0,83 \\
\hline \multirow{2}{*}{ Tamanho } & Coeficiente & $-3,25$ & 5,72 & $-0,07$ \\
\hline & Sig. & $0,00^{*}$ & $0,00^{*}$ & 0,95 \\
\hline \multicolumn{2}{|l|}{$\mathbf{R}^{2}$} & 0,39 & 0,41 & 0,12 \\
\hline \multicolumn{2}{|l|}{$\mathrm{R}^{2}$ Ajustado } & 0,15 & 0,17 & 0,01 \\
\hline \multicolumn{2}{|l|}{ Durbin-Watson } & 1,02 & 1,93 & 2,06 \\
\hline \multicolumn{2}{|l|}{ ANOVA } & $0,00^{*}$ & $0,00^{*}$ & 0,79 \\
\hline
\end{tabular}

Nota. Legenda: *Significância no nível de 5\%. **Significância no nível de $10 \%$.

Fonte: Dados da pesquisa (2018).

$\mathrm{Na}$ Tabela 6 observa-se, a partir dos modelos analisados, que o conjunto de variáveis independentes explica $39 \%$ do valor de mercado das empresas analisadas, $41 \%$ do retorno sobre os ativos e $12 \%$ do retorno sobre o patrimônio líquido, ou seja, o desempenho econômicofinanceiro. A baixa capacidade de explicação do modelo a respeito das empresas familiares e não familiares e seu desempenho também foi encontrada em estudos anteriores (Martínez et al., 2007; Bonilla et al., 2010; Din \& Javid, 2011; Shyu, 2011; Ayranci, 2014; Reyna \& Encalada, 2016).

Utilizou-se $Q$ de Tobin, ROA e ROE como proxy para medir o desempenho, o queapresentou a variável empresa familiar como positiva e significativamente relacionada ao $Q$ de Tobin e ROA. Este achado apoia a constatação de Amran e Ahmad (2009), em que o Q de Tobin é uma medida que melhor explica o desempenho da empresa, uma vez que reflete o desempenho de mercado, e não o contábil. Outra explicação para o $Q$ de Tobin, ou seja, o valor contábil dos ativos menos o valor contábil do patrimônio líquido mais o valor de mercado do patrimônio, todos divididos pelo valor contábil dos ativos, ter se destacado nesta análise se pauta em Hamberg et al. (2013) e Pukthuanthong et al. (2013), que argumentam sobre o Q de Tobin ser uma medida voltada para o futuro, que visa refletir a avaliação do mercado dos ativos das empresas em relação ao valor contábil e às futuras oportunidades de crescimento da empresa. Já o ROA e ROE, por outro lado, são consideradas medidas de lucratividade e produtividade. Assim, estes resultados revelaram que as empresas familiares e não familiares possuem diferenças em seu desempenho e valor de mercado Poletti-Hughes e Williams (2017) corroboram com esta afirmação ao evidenciarem que as empresas familiares superam as não familiares em termos de valor (desempenho de mercado).

Vieira (2014) analisou como proxies para o desempenho o $Q$ de Tobin, ROA e ROE, constatando também que um dos modelos explicativos para a relação entre as variáveis independentes (empresa familiar) e o desempenho das empresas é a variável dependente $Q$ de Tobin. Além disso, os autores encontraram resultados positivos para o ROA, assim como esta pesquisa. Os resultados de Shyu (2011), Hamberg et al. (2013), Muttakin et al. (2014), Beuren et al. (2016) e Poletti-Hughes e Williams (2017) também revelaram que a empresa familiar influencia no desempenho de mercado, a partir do $Q$ de Tobin, conforme evidenciado nesta pesquisa. No estudo de Vieira (2014), a proxy para desempenho ROE também apresentou menor valor de $\mathrm{R}^{2}$, sugerindo que esta variável não é apropriada para medir o desempenho da empresa. 
Outra variável que merece destaque é o tamanho das empresas, visto que se apresentou relacionada com os desempenhos de mercado ( $Q$ de Tobin) e econômico-financeiro (ROA). Estes achados indicam que o tamanho da empresa influencia o desempenho de mercado e econômico-financeiro da empresa, estando de acordo com Martínez et al., (2007), Bonilla et al. (2010), Shyu (2011) e Hiebl et al. (2015). Saleh et al. (2017) também encontraram uma relação significante entre o tamanho da empresa e o desempenho desta medido pelo ROE, corroborando com os achados deste estudo com empresas familiares, embora estes sejam inconsistentes com os de Anderson e Reeb (2003), que indicam a variável tamanho como não determinante do desempenho de uma empresa.

Por outro lado, nesta análise conjunta das empresas familiares e não familiares, os estágios do ciclo de vida de nascimento e crescimento se relacionaram com as proxies de desempenho, embora as demais fases (maturidade, turbulência e declínio) não tenham apresentado relações. Estes resultados não corroboram com as inferências de Dickinson (2011) e Costa et al. (2017), pois abordam que a experiência e expertise adquiridas ao longo das fases do ciclo de vida auxiliam para melhores tomadas de decisões, consequentemente melhorando 0 desempenho.

No geral, as evidências encontradas não fornecem resultados suficientes para as demais proxies de desempenho (ROE), indicando que a evidência relativa ao desempenho da empresa familiar é sensível às diferentes definições de desempenho, conforme abordado por Vieira (2014).

De modo abrangente, denota-se que as empresas familiares se diferem das demais empresas principalmente no que diz respeito a seu valor. Conforme Shyu (2011), em comparação com os outros acionistas, os membros de uma família possuem mais informações internas e podem prever as perspectivas da empresa com mais facilidade. Isto os permite tomar decisões mais precisas sobre redução ou aumento de suas propriedades, o que afeta o desempenho da empresa (Pukthuanthong et al., 2013). Além disso, as famílias podem conhecer melhor o mercado, bem como a empresa, devido a sua presença de longo prazo nesta (Pukthuanthong et al., 2013), garantindo assim um monitoramento mais eficaz e incentivando o aumento a riqueza da família, consequentemente o desempenho da empresa (Muttakin et al., 2014).

Portanto, como consequência das particularidades inerentes das empresas familiares, vários argumentos são apresentados para determinar seu desempenho destas, detectando-se assim divergências na literatura. Esses resultados conflitantes são produtos dos diferentes conceitos de empresa familiar, bem como do uso de diferentes indicadores para aferir o desempenho organizacional, afirmação corroborada pelos resultados deste estudo. Assim, sugere-se que a classificação das empresas familiares utilizadas neste estudo podem ser 0 motivo da não significância em relação à variável $Q$ de Tobin e, ao mesmo tempo, significância com, por exemplo, a variável ROA. De acordo com a literatura de empresa familiar revisitada, não foram encontrados estudos que respaldassem o motivo pelo qual nossos resultados entre as medidas de desempenho ROA, ROE e $Q$ de Tobin divergiram.

\section{CONCLUSÕES}

Este estudo teve como objetivo verificar o efeito da gestão familiar na avaliação de desempenho de 100 empresas brasileiras de capital aberto pertencentes ao setor de consumo cíclico listadas na B3. Para tanto, realizou-se uma pesquisa descritiva, documental e com abordagem quantitativa por meio da utilização da Regressão Linear Múltipla, que compreendeu os anos de 2012 a 2016. Os resultados foram analisados por meio de testes estatísticos como: teste de normalidade; correlação de Spearman, para verificar a intensidade e o sentido das correlações entre as variáveis; e Regressão Linear Múltipla, para verificar a significância dos modelos.

Os achados revelaram que as empresas familiares e não familiares diferem em seu valor, ou seja, no desempenho contábil medido pela proxy ROA, visto que a variável "empresas familiares" se relacionou significativa e positivamente com a medida de desempenho. Deste modo, na utilização do ROA, as gestões familiares influenciaram positivamente o desempenho 
da empresa, diferentemente do ocorrido para o desempenho contábil medido pelo ROE e desempenho de mercado pelo $Q$ de Tobin, visto que estas variáveis não apresentaram relação com as empresas familiares. Outra variável de destaque no modelo analisado foi o tamanho da empresa, que influencia no desempenho da amostra analisada. Para tanto, o tamanho da empresa implica em maior eficiência no uso de ativos pelas empresas familiares do setor cíclico, o que denota uma relação positiva com os desempenhos de mercado e contábil.

Estes resultados permitem inferir por que as empresas familiares são um dos mais importantes modelos de desenvolvimento de negócios no Brasil e em outros países, além de mostrarem uma relação no desempenho das empresas familiares e não familiares. Assim, um aumento na propriedade familiar pode desencadear um aumento no desempenho de mercado, o que indica que a família tem um incentivo mais forte para maximizar o desempenho da empresa, por sua riqueza se atrelar a ela.

Infere-se, portanto, que há em empresas familiares um engajamento maior no intuito da obtenção de melhor desempenho contábil. Estas valorizam a maximização do valor da empresa a longo prazo, o que também foi comprovado por estudos anteriores (Bonilla et al., 2010; Shyu, 2011; Hiebl et al., 2015). Segundo Shyu (2011), Ayranci (2014) e Reyna e Encalada (2016), estes achados demonstram que há diferenças de desempenho entre empresas familiares e não familiares.

Este estudo contribui cientificamente no que tange às finanças corporativas, tanto pela identificação de elementos de desempenho das empresas comumente utilizados, assim como por agregar à literatura existente a realidade das organizações familiares brasileiras do setor de consumo cíclico. Além disso, esta pesquisa pode contribuir socialmente ao colaborar com o mercado de capitais na avaliação de mercado das companhias listadas no setor de consumo cíclico na bolsa de valores. Além disso, este estudo fornece um panorama de diferentes proxies que podem ser adotadas pelas empresas familiares para avaliação de desempenho, com vistas a buscarem um diferencial no mercado de capitais, visto que a saúde financeira das empresas pode atrair investidores. A contribuição desta pesquisa pode ser identificada também em relação à maior eficiência dos gestores quanto ao desempenho contábil, dado que as empresas desta pesquisa apresentaram diferenças no desempenho de mercado medido pela proxy ROA.

Este trabalho, apesar de suas contribuições acerca do efeito da gestão familiar na avaliação do desempenho de empresas do setor de consumo cíclico, apresenta limitações. A primeira consiste na utilização de somente um setor da B3, impossibilitando a generalização dos resultados para outros setores. Em segundo lugar, a análise de apenas empresas brasileiras impossibilita a comparabilidade dos resultados com outros países. Outra limitação diz respeito ao modelo pesquisado, visto que outras variáveis de desempenhos de mercado, econômicofinanceiro e outros determinantes do desempenho de empresas familiares e não familiares poderiam ter sido utilizadas. Ainda, outros fatores como variáveis contingenciais e cultura organizacional, juntamente com o contexto de empresa familiar, podem afetar o desempenho de uma organização.

Sugere-se para investigações futuras o aumento da amostra, tanto para outros setores econômicos da Bolsa de Valores como para outros países, a fim de obter um quadro mais amplo da realidade das empresas familiares brasileiras e possibilitar a comparabilidade dos resultados, bem como sua generalização para economias semelhantes. Recomenda-se também a replicação deste estudo em outros países para a consolidação da literatura sobre empresas familiares. Como a influência familiar é um tema relevante e que desperta o interesse da academia, a análise de seu impacto sobre os negócios financeiros torna-se necessária por meio de outras variáveis. Sugere-se também considerar o processo sucessório e a migração da gestão familiar para a profissional. Portanto, novas pesquisas com abordagens mais aprofundadas sobre o tema são essenciais.

\section{REFERÊNCIAS}

Acquaah, M. (2013). Management control systems, business strategy and performance: a comparative analysis of Family and non-family business in a transmition economy in subSaharan Africa. Journal of Family Business Strategy,4(2), 131-146. 
Amran, N. A. \& Ahmad, A. C. (2009). Family business, board dynamics and firm value: Evidence from Malaysia. Journal of Financial Reporting and Accounting, 7(1), 53-74.

Anderson, R. C., \& Reeb, D. M. (2003). Founding-family Ownership and Firm Performance: Evidence for the S\&P 500. The Journal of Finance, 58(3), 1301-1328.

Andres, C. (2008). Large shareholders and firm performance: an empirical examination of founding-family ownership. Journal of Corporate Finance, 14(4), 43-445.

Ayranci, E. (2014). A study on the influence of family on family business and its relationship to satisfaction with financial performance. Business Administration and Management, 17(2), 87-105.

Berrone, P., Cruz, C., \& Gomez-Mejia, L. R. (2012). Socioemotional wealth in family firms: Theoretical dimensions, assessment approaches, and agenda for future research. Family Business Review, 25(3), 258-279.

Beuren, I. M., Hein, N., \& Boff, M. L. (2011). Estratégias de legitimidade organizacional de Lindblom (1994) versus geração familiar gestora de empresas familiares. Revista Iberoamericana de Contabilidad de Gestión, 9(17), 1-20.

Beuren, I. M., Politelo, L., \& Martins, J. A. S. (2013). Influência da propriedade familiar no desempenho das empresas da BM\&FBovespa. Anais da Associação Nacional de Programas de Pós-Graduação em Ciências Contábeis, Fortaleza, CE, Brasil, 7.

Beuren, I. M., Politelo, L., \& Martins, J. A. S. (2016). Influence of family ownership on the companies performance. International Journal of Managerial Finance, 12(5), 1-36.

Bonilla, C. A., Sepulveda, J., \& Carvajal, M. (2010). Family ownership and firm performance in Chile: A note on Martinez et al.'s evidence. Family Business Review, 23(2), 148-154.

Burkart, M., Panunzi, F., \& Shleifer, A. (2003). Family firms. The Journal of Finance, 58(5), 21672201.

Chahine, S. (2007). Block-holder ownership, family control and post-listing performance of French IPOs. Managerial Finance, 33(6), 388-400.

Chua, J. H., Chrisman, J. J., \& Sharma, P. (1999). Defining the Family business by behavior. Entrepreneurship: theory and practice, 23(4), 19-19.

Costa, W. B. da, Macedo, M. A. S., Yokoyama, K. Y., \& Almeida, J. E. F. de. (2017). Análise dos Estágios de Ciclo de Vida de Companhias Abertas no Brasil: Um Estudo com Base em Variáveis Contábil-Financeiras. Brazilian Business Review, 14(3), 304-320.

Dawson, A. \& Mussolino, D. (2014). Exploring what makes Family firms diferente: discrete or overlapping constructs in the literature? Jounal of Family Business Strategy, 5, 169-183.

Dickinson, V. (2011). Cash flow pattems as a proxy for firm life cycle. The Accounting Review, 86(6),1969-1994.

Din, S. U. \& Javid, A. Y. (2011). Impact of managerial ownership on financial policies and the firm's performance: evidence Pakistani manufacturing firms.

Donckels, R., \& Fröhlich, E. (1991). Are Family business really diferente? European Experiences from stratos. Family Business Review, 4(2), 1-15.

Erbetta, F., Menozzi, A., Corbetta, G., \& Fraquelli, G. (2013). Assessing Family firm performance using frontier analysis techniques: evidence from Italian manufacturing industries. Journal of Family Business Strategy, 4(2), 106-117.

Family Firm Institute - FFI. (2015). Recuperado em 25 abril, 2018, de http://www.ffi.org/ 
Fávero, L. P., Belfiore, P., Silva, F. D., \& Chan, B. L. (2009). Análise de dados: modelagem multivariada para tomada de decisões.

Fávero, L. P. (2015). Análise de Dados: Modelos de Regressão com Exce॥, STATA® e SPSS $\AA$. Rio de Janeiro: Elsevier.

Franks, J., Mayer, C., Volpin, P., \& Wagner, H. F. (2012), The life cycle of Family ownership: international evidence. The Review of Financial Studies, 25(6), 1675-1712.

Frezatti, F., Bido, D. S., Mucci, D. M., \& Beck, F. (2017). Estágios do ciclo de vida e perfil de empresas familiares brasileiras. Revista de Administração de Empresas, 57(6), 601-619.

Gómez-Mejía, L. R., Haynes, K. T., Núñez-Nickel, M., Jacobson, K. J. L., \& Moyano-Fuentes, J. (2007). Socioemotional Wealth and Business Risks in Family-controlled firms: Evidence from Spanish Olive Oil Mills. Cidade Universitária, 31(1), 106-137.

Gujarati, D. N., \& Porter, D. C. (2006). Dasar-dasar ekonometrika. Jakarta: Erlangga.

Halili, E., Saleh, A. S., \& Zeitun, R. (2015). Governance and long-term operating performance of family and non-family firms in Australia. Studies in Economics and Finance, 32(4), 398-421.

Hamberg, M., Fagerland, A. E., \& Nilsen, K. K. (2013). Founding-family firms and the creation of value: Swedish evidence. Managerial Finance, 39(10), 963-978.

Hiebl, M. R. W., Duller, C., Feldbauer-Durstmüller, B., \& Ulrich, P. (2015). Family influence and management accounting usage - findings from Germany and Austria. Schmalenbach Business Review, 67(3), 368-404.

Holt, D. T., Rutherford, M. W., \& Kuratko, D. F. (2010). Advancing the field of Family business research: further testing the measurement properties of the F-PEC. Family Business Review, 23(1), 76-88.

Kang, H. C. \& Kim, J. (2016). Why do family firms switch between family CEOs and non-family professional CEO? Evidence from Korean Chaebols. Review of Accounting and Finance, 15(1), 45-64.

Macedo, M. A. S. \& Corrar, L. J. (2010). Analise do desempenho contábil-financeiro de seguradoras no Brasil no ano de 2007: um estudo apoiado em análise hierárquica (AHP). Contabilidade Vista \& Revista, 21(3), 135-165.

Martínez, J. I., Stöhr, B. S., \& Quiroga, B. F. (2007). Family ownership and firm performance: Evidence from public companies in Chile. Family Business Review, 20(2), 83-94.

Masri, T. E., Tekathen, M., Magnan, M., \& Boulianne, E. (2017). Calibrating management control technologies and the dual identity of family firms. Qualitative Research in Accounting \& Management, 14(2), 157-188.

Maury, B. (2006). Family ownership and firm performance: empirical evidence from Western European corporations. Journal of Corporate Finance, 12(2), 321-341.

Mazzi, C. (2011). Family business and financial performance: current state of knowledge and future research challenges. Journal of Family Business Strategy, 2(3), 166-181.

Micheli, P. \& Mura, M. (2017). Executing strategy through comprehensive performance measurement systems. International Journal of Operations \& Production Management, 37(4), 423-443.

Muttakin, M. B., Khan, A., \& Subramaniam, N. (2014). Family firms, family generation and performance: evidence from an emerging economy. Journal of accounting in emerging economies, 4(2), 197-219.

Olson, E. M. \& Slater, S. F. (2002). The balanced scorecard, competitive strategy, and performance. Business Horizons, 45(3), 17-24. 
Poletti-Hughes, J. \& Williams, J. (2017). The effect of Family control on value and risk-taking in Mexico: a sociomotional wealth approach. International Review of Financial Analysis.

Politelo, L., Kaveski, I. D. S., Chiarello, T. C., \& Silva, T. P. (2014). Propriedade Familiar e Remuneração Baseada em Ações da Diretoria das Empresas Familiares. Revista Ambiente Contábil, 6(1), 183-201.

Pukthuanthong, K., J. Walker, T., Thiengtham, D. N., \& Du, H. (2013). Does family ownership create or destroy value? Evidence from Canada. International Journal of Managerial Finance, 9(1), 13-48.

Reyna, J. M. S. M. \& Encalada, J. A. D. (2016). Sucesión y su relación com endeudamiento y desempeño en empresas familiares, 61(1), 41-57.

Saleh, A. S., Halili, E., Zeitun, R., \& Salim, R. (2017). Global financial crisis, ownership structure and firm financial performance: An examination of listed firms in Australia. Studies in Economics and Finance, 34(4), 447-465.

Sciascia, S., Mazzola, P., \& Kellermanns, F. W. (2014). Family management and profitability in private family-owned firms: introducing generational stage and the socioemotional wealth perspective. Journal of Family Business Strategy, 5(2), 131-137.

Sharma, P. (2004). An overview of the field of Family business studies: current status and directions for the future. Family Business Review, 17(1), 1-36.

Sharma, P., Chrisman, J. J., \& Chua, J. H. (1997). Strategic management of the Family business: past research and future challenges. Family Business Review, 10(1), 1-35.

Shyu, J. (2011). Family ownership and firm performance: evidence from Taiwanese firms. International Journal of Managerial Finance, 7(4), 397-411.

Speckbacher, G. \& Wentges, P. (2012). The impact of Family control on the use of performance measures in strategic target setting and incentive compensation: a research note. Management Accounting Research, 23(1), 34-46.

Ussman, A. M. (1996). As empresas familiares - características e problemática. Estudos de Gestão, 3(1), 19-26.

Vieira, E. F. S. (2014). The effect on the performance of listed family and non-family firms. Managerial Finance, 40(3), 234-253.

Wooldridge, J. M. (2012). Introdução à Econometria: uma abordagem moderna. São Paulo: Cengage Learning.

Zahra, S. A. \& Sharma, P. (2004). Family business research: A strategic reflection. Family Business Review, 17(4), 331-346.

Zahra, S. A. (2005). Entrepreneurial risk taking in family firms. Family Business Review, 18(1), 23-40. 